\title{
Refractive index-enhanced vortex lattices
}

O. E. Mustecaplioglu

M. O. Oktel 


\title{
Refractive index-enhanced vortex lattices
}

\author{
Ö. E. Müstecaplıoğlu ${ }^{a}$ and M. Ö. Oktel ${ }^{b}$ \\ ${ }^{a}$ Department of Physics, Koç University, Sarıer, Istanbul, 34450, Turkey; \\ ${ }^{b}$ Bilkent University, Department of Physics, 06800 Bilkent, Ankara, Turkey
}

\begin{abstract}
Light propagation through vortex matter in atomic Bose-Einstein condensates is examined. It is shown that vortex matter can be used as a photonic crystal by a refractive index enhancement scheme. Band structure of the vortex lattice is numerically calculated. Index enhanced vortex matter is shown to exhibit large refractive index contrast with the dilute thermal gas background in the vortex core. Depending on the depth of the index contrast full or directional photonic band gaps are found in the band structure. Experimental parameters required to generate band gaps in the visible region of the electromagnetic spectrum are calculated.
\end{abstract}

Keywords: Bose-Einstein condensates, vortex lattice, photonic crystals, refractive index enhancement

\section{INTRODUCTION}

In-situ optical probing of vortices in rotating atomic Bose-Einstein condensates (BECs) is a challenging task due to the small size of vortices requiring imaging resolution close to a single wavelength. It is usually necessary to let the condensate expand ballistically before employing imaging methods. ${ }^{1-5}$ In-situ imaging of the vortex lattice has been demonstrated, by imaging the cloud perpendicular to the axis of rotation and observing patterns created by the overlapping lattice planes. ${ }^{6}$ Alternative schemes based upon ultra-slow light imaging have been proposed for non-destructive, in-situ dynamical imaging of topological and collective excitations ${ }^{7}$ as well as for probing phase evolution of the condensate. ${ }^{8}$

In a simple treatment of light propagation in a vortex lattice of a BEC, one may ignore the spatial periodicity in the system as the vortex cores and the atomic sample would have negligible difference in their refractive indices. The present paper however, aims to exploit this periodicity to generate photonic band gaps, similar to those in photonic crystals which are well-known for their light localization effects. ${ }^{9,10}$ By modifying the dispersion relation of light, photonic crystals create many optical effects ranging from left-handed light propagation ${ }^{11}$ to slowing down or stopping light. ${ }^{12,13}$ If the vortex lattice of the condensate may be utilized as a photonic crystal, these optical effects can be used to modify or probe the condensate, or create novel atom-optical devices.

As a first example, presence of a photonic band gap may provide stability to the vortex lattice against radiative losses. ${ }^{14}$ Such losses are especially problematic as a source of decoherence in the quantum information applications, based upon generation, storage and transfer of nonclassical states in atom-optical systems. ${ }^{15-19}$ These operations typically involve excited states and would suffer from spontaneous emission. The idea of suppressing spontaneous emission and enhancing stability of a Bose condensed system using photonc band gaps was first discussed for the case of optical lattices. ${ }^{20}$ Unfortunately, due to the competing effects of weak excitations and the strong periodic potential which are both provided by the same laser beams forming the optical lattice, the suppression was found to be insignificant. ${ }^{20}$ In the case of a vortex lattice, however, the periodicity is a topological effect and the atoms can be excited by a completely different and independent physical system such as a pump laser. Hence, one would expect to achieve a more significant suppression in the case of vortex lattices, which could be helpful to improve performance of atom-optical devices. One may also exploit the photonic band gap as an alternative to the existing EIT schemes for slowing down or stopping light, as discussed in a number of recent papers. ${ }^{12}$

Further author information: (Send correspondence to Ö.E.M.)

Ö.E.M.: E-mail: omustecap@ku.edu.tr, Telephone: +90 (212) 3381424

M.Ö.O.: E-mail: oktel@fen.bilkent.edu.tr, Telephone: +90 (312) 2902512 


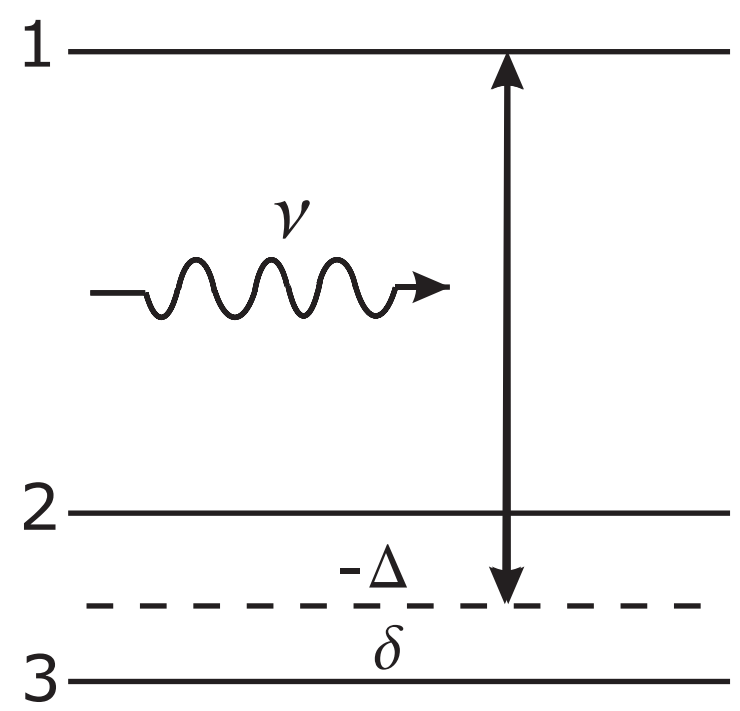

Figure 1. Three-level atom interacting with a field of frequency $\nu$. The optical field detuned from the resonant transitions by $\Delta=\omega_{12}-\nu$ and $\delta=\omega_{13}-\nu$.

In this letter, we discuss presence of photonic band gaps in a vortex lattice and show how these band gaps can be used to measure the rotation frequency of a vortex lattice. In vortex lattice experiments, the rotation rate of the vortex lattice is not directly measured, but rather inferred from the change of the cloud's aspect ratio upon rotation. ${ }^{1-5}$ It is assumed that, with a fully developed vortex lattice, the cloud rotates like a solid body; on the other hand coupling of the collective modes of the lattice with the cloud's collective oscillations may result in a much more complicated behavior. An independent measurement of the rotation frequency of the lattice would clarify the dependence of the cloud aspect ratio on rotation as well as determine clearly how far one can probe into the lowest Landau level regime. ${ }^{21}$

\section{INDEX ENHANCED VORTEX LATTICE}

In order to have a significant photonic band gap, it is preferable to have a material consisting of periodically alternating sequence of dielectric domains with high contrast in their dielectric constants. Dilute atomic gases exhibit strong differences from the dielectric constant of free space only at their resonance frequencies, where the optical fields would suffer high absorbance. To overcome this challenge, we consider utilization of a quantum coherent index enhancement scheme which allows for many orders of magnitude improvement in the refractive index of an atomic gas at frequencies where the absorption can be made zero. ${ }^{22}$ This effect is based upon the similar principle of quantum coherence in electromagnetically induced transparency (EIT), ${ }^{23}$ which is used for slow-light ${ }^{24}$ and frozen light effects. ${ }^{25}$

There are various schemes in which index enhancement accompanied by vanishing absorption is possible, such as, initial coherence scheme, Raman driven scheme, and microwave driven schemes. ${ }^{26,27}$ The text book result for the optical response of an atom with the level structure depicted in Fig.1 is known to be, ${ }^{26}$

$$
\begin{aligned}
\chi^{\prime}= & -\frac{d^{2} N}{\epsilon_{0} \hbar \gamma_{1}}\left\{\frac { 1 } { \gamma _ { 1 3 } ^ { 2 } + \Delta ^ { 2 } } \left[\Delta\left(\frac{\rho_{11}^{(0)}}{\gamma_{1}}-\frac{\rho_{33}^{(0)}}{\gamma_{3}}\right)\right.\right. \\
& \left.-\frac{\left|\rho_{32}^{(0)}\right|}{\sqrt{\gamma_{32}^{2}+\omega_{32}^{2}}}\left(\Delta \cos \phi+\gamma_{13} \sin \phi\right)\right]
\end{aligned}
$$




$$
\begin{aligned}
& +\frac{1}{\gamma_{12}^{2}+\delta^{2}}\left[\delta\left(\frac{\rho_{11}^{(0)}}{\gamma_{1}}-\frac{\rho_{22}^{(0)}}{\gamma_{2}}\right)\right. \\
& \left.-\frac{\left|\rho_{32}^{(0)}\right|}{\sqrt{\gamma_{32}^{2}+\omega_{32}^{2}}}\left(\delta \cos \phi-\gamma_{12} \sin \phi\right)\right], \\
\chi^{\prime \prime}= & -\frac{d^{2} N}{\epsilon_{0} \hbar \gamma_{1}}\left\{\frac { 1 } { \gamma _ { 1 3 } ^ { 2 } + \Delta ^ { 2 } } \left[\gamma_{13}\left(\frac{\rho_{11}^{(0)}}{\gamma_{1}}-\frac{\rho_{33}^{(0)}}{\gamma_{3}}\right)\right.\right. \\
& \left.-\frac{\left|\rho_{32}^{(0)}\right|}{\sqrt{\gamma_{32}^{2}+\omega_{32}^{2}}}\left(\gamma_{13} \cos \phi-\Delta \sin \phi\right)\right] \\
& +\frac{1}{\gamma_{12}^{2}+\delta^{2}}\left[\gamma_{12}\left(\frac{\rho_{11}^{(0)}}{\gamma_{1}}-\frac{\rho_{22}^{(0)}}{\gamma_{2}}\right)\right. \\
& \left.-\frac{\left|\rho_{32}^{(0)}\right|}{\sqrt{\gamma_{32}^{2}+\omega_{32}^{2}}}\left(\gamma_{12} \cos \phi+\delta \sin \phi\right)\right],
\end{aligned}
$$

where $\Delta=\omega_{13}-\nu, \delta=\omega_{12}-\nu$, and the phase $\phi$ is defined by $\phi=\arg \left[\rho_{32}^{(0)}\right]+\tan ^{-1}\left(\omega_{32} / \gamma_{32}\right)$. For simplicity, electric dipole moment matrix elements $d_{12}=d_{13}=d_{21}=d_{31} \equiv d$ have been used.The atomic coherence is represented by $\phi$. In the treatment of ultra-slow light propagation, the optical response is assumed to be local such that the atomic density $N=N(\vec{r})$ was directly used in $\chi$ and we have $\chi=\chi(\nu, \vec{r})$. As an estimate of $\chi^{\prime}$ at the laser frequency where $\chi^{\prime \prime}=0$ and $\chi^{\prime}$ is maximum, one can write ${ }^{27}$

$$
\chi^{\prime} \sim \frac{1}{10} \frac{d^{2} N}{\hbar \epsilon_{0} \gamma_{1}},
$$

which can be expressed in a more convenient form by using the relation

$$
\gamma=\frac{d^{2} \nu^{3}}{6 \pi \hbar \epsilon_{0} c^{3}}
$$

that yields,

$$
\chi^{\prime} \sim \frac{1}{10} \frac{3 N \lambda^{3} \gamma}{4 \pi^{2} \gamma_{1}}
$$

We Assume purely radiative decay so that $\gamma_{1}=\gamma$. Here $N$ is the upper level atomic concentration. Higher index contrast can be achieved using upper-level microwave scheme which is illustrated with the energy level diagram in the inset of Fig. 2. Quantum coherence is established through coupling of levels $a$ and $c$ via a strong microwave field, which allows for absorption free propagation of a probe field dipole coupled to levels $a$ and $b$. A pump mechanism from level $b$ to $c$ ensures an ultra high refractive index by maintaining a small fraction of atoms in level $c$. At the point of vanishing absorption, susceptibility $(\chi)$ becomes a real number and can be estimated to be

$$
\chi \sim 3 N \lambda^{3} / 40 \pi^{2},
$$

where $N$ is now the atomic density, and $\lambda$ is the wavelength of $a-b$ transition. In a dense BEC, the first correction to the susceptibility is argued to be the local field correction, ${ }^{28-32}$ which changes $\chi$ to a corrected value, ${ }^{33}$

$$
\chi \rightarrow \frac{\chi}{\left(1-\frac{\chi}{3}\right)}
$$

Local field corrections are small below $N \sim 10^{21} \mathrm{~m}^{-3}$ at which $\chi$ increases to 3.22 from 1.55 for ${ }^{23} \mathrm{Na}$ gas of $\lambda=589 \mathrm{~nm}$. For ${ }^{87} \mathrm{Rb}$ gas of $\lambda=794 \mathrm{~nm}, N=5.5 \times 10^{20} \mathrm{~m}^{-3}$, we find $\chi \sim 7$ including the local field correction. 

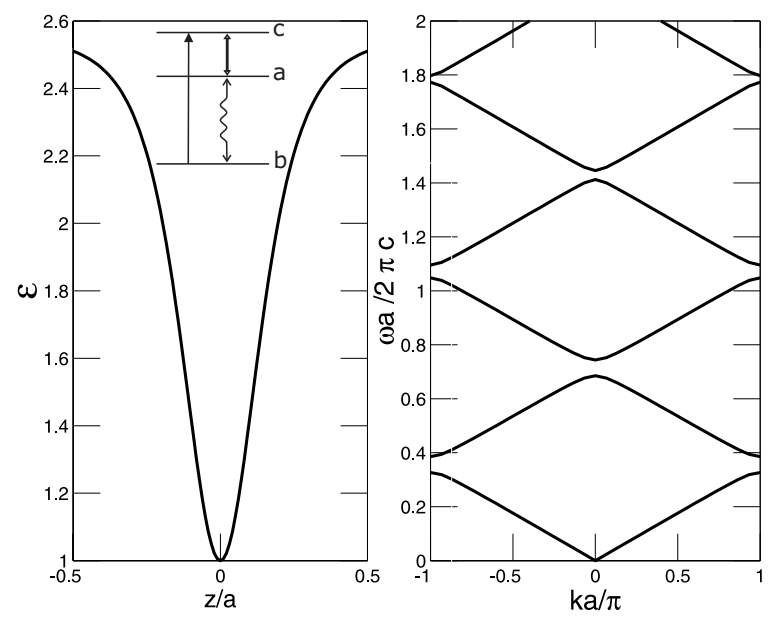

Figure 2. (Left) Position dependence of the dielectric function of the one dimensional vortex lattice within a spatial period where the background dielectric constant is assumed to be $\epsilon_{0}=1$. Inset shows the level scheme of the upper-level microwave driven scheme. (Right) Band structure of a one dimensional vortex lattice.

The dispersive behavior of $\epsilon$ near the frequency of index enhancement will be ignored. This seems reasonable as $\chi$ is slowly varying about its peak value where the first derivative of $\chi$ vanishes and the first correction is of second order in probe detuning.

As our atomic medium, we consider a vortex lattice of rotating BEC. It has been predicted, ${ }^{21,34-36}$ and observed $^{1-5,37}$ that the vortex lattice is triangular. It has recently been shown that triangular lattice is robust under variations of the trap potential in two dimensions. ${ }^{38}$ The number of vortices can be as large as few hundred. Presently, we assume the lattice is large and ignore finite size effects. Lattice spacing $a$ can be compared to the healing length

$$
\xi=\frac{1}{\sqrt{8 \pi N a_{s c}}}
$$

where $a_{s c}$ is the scattering length. We consider $a \sim 5-10 \xi$. Our examinations indicate a rich variation of band structure depending on $a$. Below, we report photonic band gaps in the visible range.

\subsection{Photonic Band Gaps of Vortex Rows}

To study the effects of the vortex profile on the band structure, we consider a simpler system of one dimensional vortex lattice. Such vortex row structures have theoretically been predicted, ${ }^{38,39}$ but have not been observed so far. We use a vortex profile $\rho(z)$ in the Padé approximation. ${ }^{40}$ single straight line vortex can be described by a profile as

$$
\rho(r)=\frac{r^{2}\left(a_{1}+a_{2} r^{2}\right)}{1+b_{1} r^{2}+b_{2} r^{4}}
$$

with

$$
\begin{aligned}
& a_{1}=0.3437, \quad b_{1}=\frac{5-32 a_{1}}{48-192 a_{1}}, \\
& a_{2}=a_{1}\left(b_{1}-0.25\right), \quad b_{2}=a_{2} .
\end{aligned}
$$

Our calculations show that the form of vortex profile causes little change in the band structure. It is convenient and efficient to have an analytical form for the dielectric function (particularly its Fourier transform) in numerical 


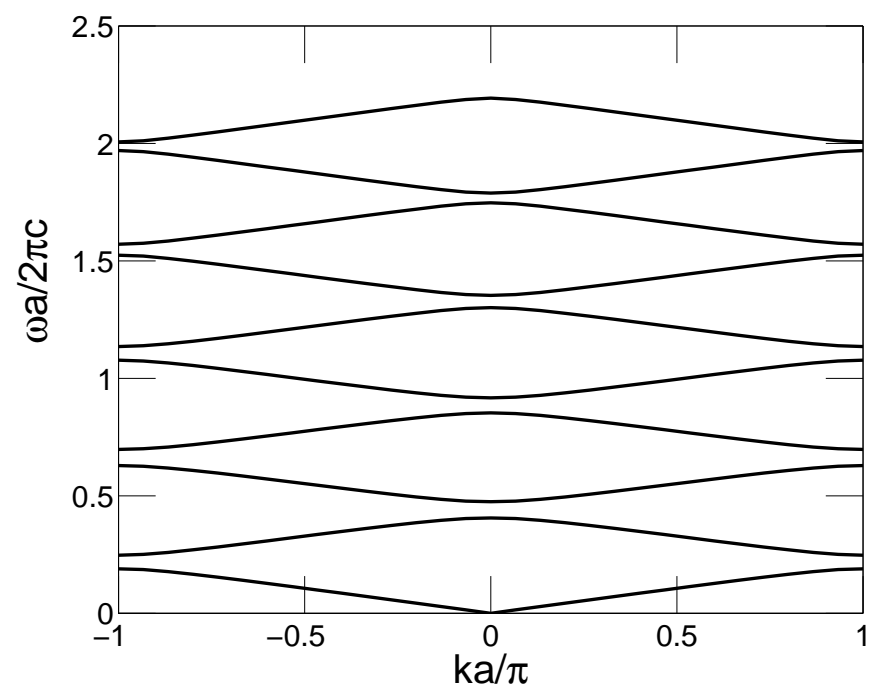

Figure 3. Band structure of a one dimensional vortex lattice of ${ }^{87} \mathrm{Rb}$ with $N=5.5 \times 10^{20} \mathrm{~m}^{-3}$ and $a=10 \xi$.

calculation of the band structures. In the dilute gas limit, The background thermal gas component is assumed to be dilute in the vortex core regions and we further assume that it has almost uniform density. We can therefore take the dielectric constant within the vortex core region is approximately same with that of free space value, $\epsilon_{0}=1$. Using $\epsilon_{1}=1+\chi^{\prime}$, dielectric function of the atomic system can be expressed in the form

$$
\epsilon(z)=\epsilon_{0}+\left(\epsilon_{1}-\epsilon_{0}\right) \rho(z)=1+\chi^{\prime} \rho(z) .
$$

We calculate the band structure of the vortex lattice of $N=6.6 \times 10^{20} \mathrm{~m}^{-3}{ }^{23} \mathrm{Na}$ atoms using plane wave expansion method with $11 \times 11$ grid of plane waves. Our results for $a=10 \xi$ are shown in Fig.2. There is a full gap at $\omega a / 2 \pi c=0.35$ with a gap-midgap ratio of 0.143 . In a similar band structure for ${ }^{87} \mathrm{Rb}$, with $N=5.5 \times 10^{20} \mathrm{~m}^{-3}$, full gaps at visible midgap frequencies $520 \mathrm{THz}$ and $445 \mathrm{THz}$ are found when $a=10 \xi$ and $a=5 \xi$, respectively. Results for the case of $a=10 \xi$ are shown in Fig.3 and in Fig.4.

\subsection{Photonic Band Gaps of Triangular Vortex Lattice}

In the two-dimensional geometry, we consider straight line vortices with perfect cylindrical symmetry for simplicity. For vortices as effective cylinders of radius $R=2 \xi$, this corresponds to a filling ratio $f=(2 \pi / \sqrt{3})\left(R^{2} / a^{2}\right) \sim$ 14\%. Typical results for TE polarization are shown in Fig.5 for the two-dimensional lattice. Similar band structure occurs for TM polarization. In the TM case, the gap between the first and second bands decreases and becomes negligibly small at the $K$ point of the Brillouin zone. For the TE case, a gap appears at all points in the irreducible Brillouin zone including the $M$ point. There are directional pseudogaps but no complete band gap for both TM and TE polarizations.

It should be emphasized that it is actually possible to observe full band gap, as it is typical for triangular lattice, for another choice of parameters. Assuming $a>\sim 4 \xi$ gives $R / a<\sim 0.5$ for the radius of the vortex core $R=2 \xi$. A full gap for TE polarization is found for ${ }^{87} \mathrm{Rb}$ with $a=4.5 \xi$ at $N=5.5 \times 10^{20} \mathrm{~m}^{-3}$. The corresponding band structures are given in Fig. 6 for the TE polarization and in Fig.7 for the TM polarization. For this case the filling ratio is about $\sim 0.72, \xi \sim 115 \mathrm{~nm}$. Including the local field correction we calculate the dielectric constant of the vortex matter is $\sim 8$ while the core region has unit dielectric constant. Full gaps for both polarizations may appear at higher index-contrasts.

\section{FINITE VORTEX LATTICE}

After investigating an infinite vortex lattice and finding that band gaps develop, we consider whether the effects discussed before can be observed in an experiment where the vortex lattice is necessarily finite. In a typical 


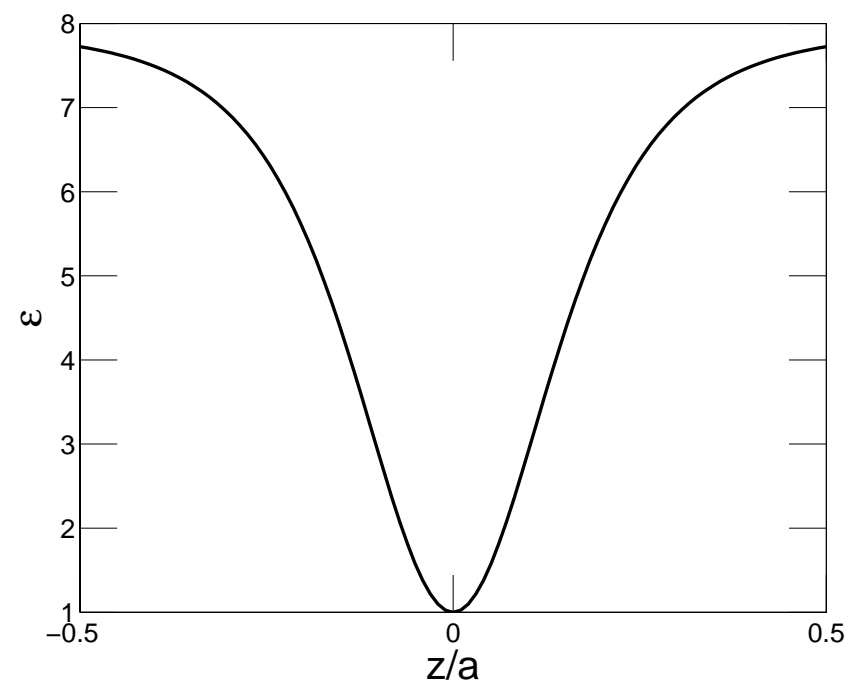

Figure 4. Same with Fig.3 but for the position dependence of the dielectric function of the one dimensional vortex lattice within a spatial period.

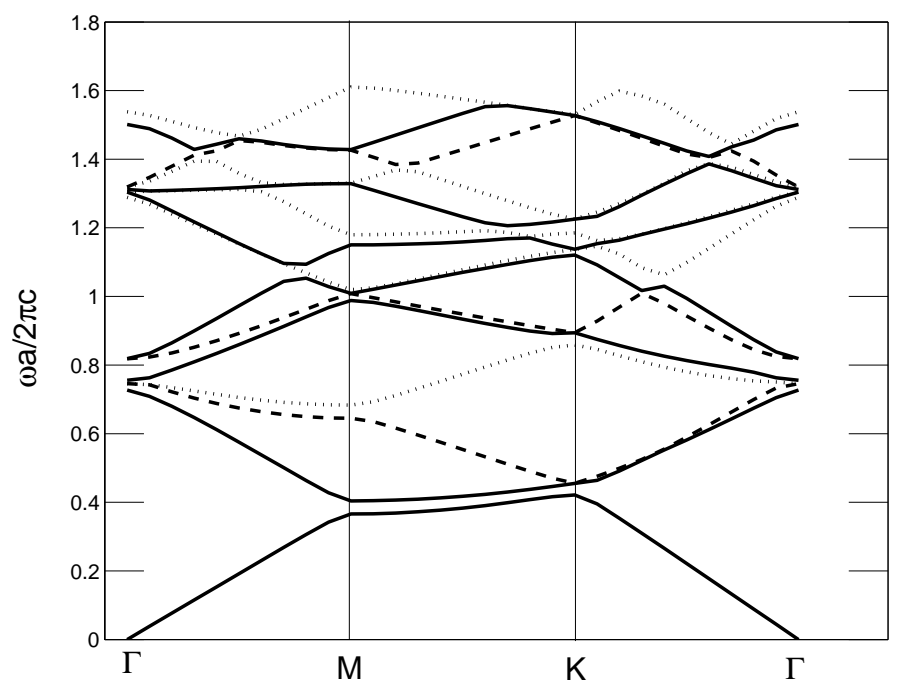

Figure 5. TE band structure of a triangular vortex lattice with $a=10 \xi$ of $N=6.6 \times 10^{20} \mathrm{~m}^{-3}{ }^{23} \mathrm{Na}$ atoms. Different line patterns are used for visual clarity. 


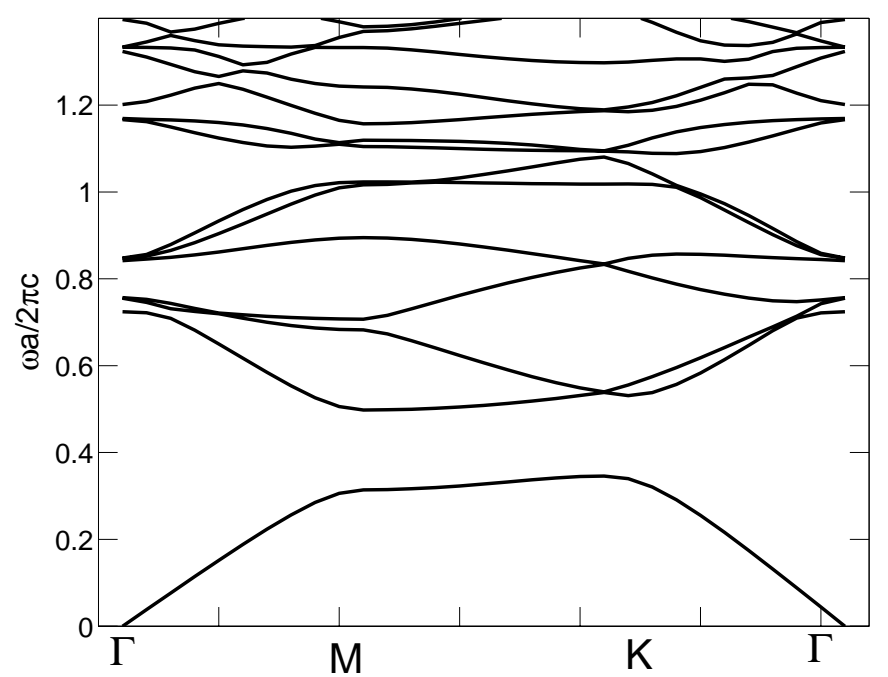

Figure 6. TE band structure of a triangular vortex lattice with $a=4.5 \xi$ of $N=5.5 \times 10^{20} \mathrm{~m}^{-3}{ }^{87} \mathrm{Rb}$ atoms $\left(a_{s c}=\right.$ $5.5 \mathrm{~nm}, \lambda=794 \mathrm{~nm})$.

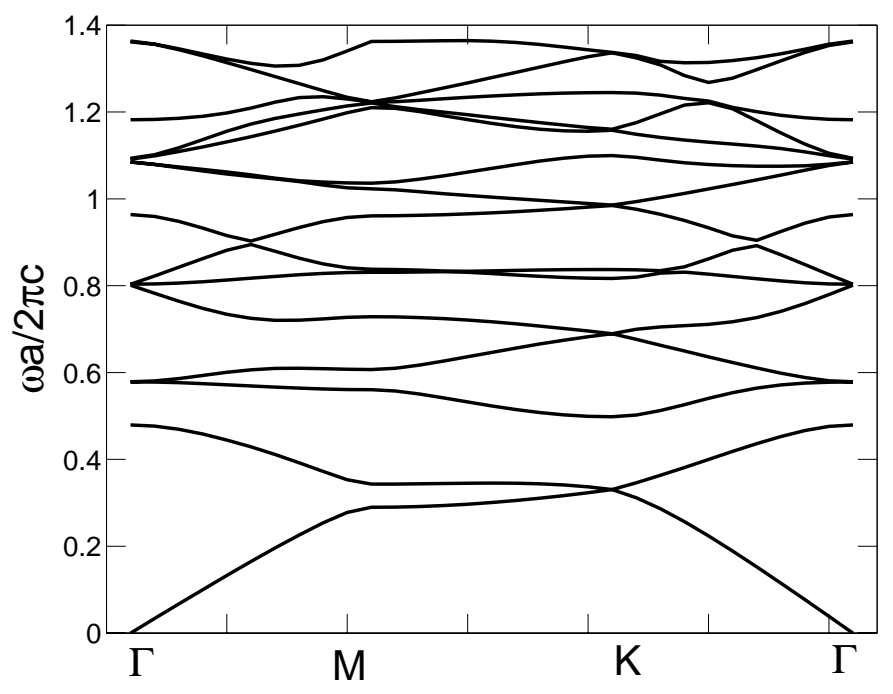

Figure 7. Same with Fig. 6 but for the TM band structure 


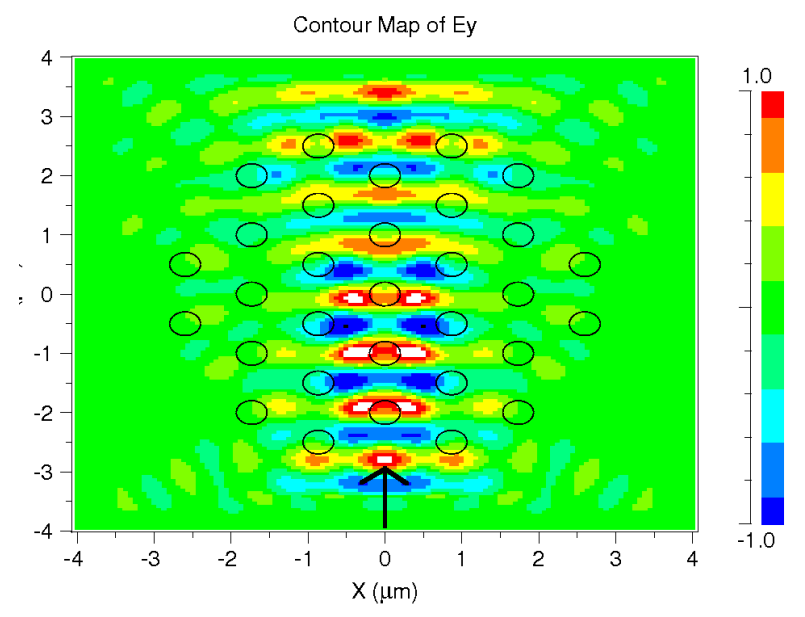

Figure 8. A vortex lattice with 31 vortices. Vortex spacing is taken to be 5 times the vortex core size which is equal to the light wavelength. Vortex cores have $n=1$ while BEC has index of refraction $n=3$. With these parameters infinite lattice results indicate a directional band gap, which can be observed by comparing this figure with the Fig.9. Direction of the incident light is denoted by an arrow in both figures.

experiment, there are three major differences from the idealized vortex lattice considered above. Here, we consider all three separately.

First, we assumed a strictly two dimensional vortex lattice, which disregards any bending or motion of the vortices along the rotation axis. However, the bending of the vortices have theoretically been predicted to be small except near the edges of the cloud. ${ }^{21,41}$ This prediction was later confirmed by viewing the vortex lattice from the side. ${ }^{6}$ As the deviation of the position of the vortices are much less than the vortex spacing, we expect the bending, or Kelvin mode excitations of the vortices in the third dimension to be a very small effect.

Second simplification in our calculation was that the index profile of the cloud remained uniform except for the appearance of vortices. In a trapped BEC with vortices overall density profile is maximum in the middle and would slowly decay towards the edges. Average density profile is predicted to be Gaussian ${ }^{21}$ or Thomas-Fermi like, ${ }^{42}$ in both cases a monotonous slowly varying function. This slow variation of the index profile would create an extra source of scattering from the cloud, especially under coherent index enhancement. The presence of this extra scattering may complicate the optical band structure effects if the variation of the density is not slow on the scale of vortex spacing. However, as the overall density decays monotonously, a fast variation of the density on the scale of vortex spacing would mean there are only a small number of vortices in the cloud. So for a BEC containing enough number of vortices to create band structure effects, photonic band structure should be superimposed on the extra scattering effect which is expected to vary slowly in frequency. Thus the photonic crystal effects should be easily observable.

A third, and related to second, concern in an experiment would be the finiteness of the vortex lattice. We performed a numerical simulation of the propagation of an electromagnetic wave in a finite lattice to quantify the number of vortices needed to observe band structure effects. In Figs.8-9, we display our results for 31 vortices in two different orientations. The simulation was done with parameters where the infinite lattice calculation indicates a directional band gap, and this band gap is clearly seen by contrasting the two figures. In Fig.8, a Gaussian beam passes through the vortex lattice without much scattering, while in Fig.9, after a rotation of the vortex lattice by $\pi / 6$, there is no transmission. Our results indicate that around 20 vortices are enough to qualitatively observe the infinite lattice predictions for the band gaps. 


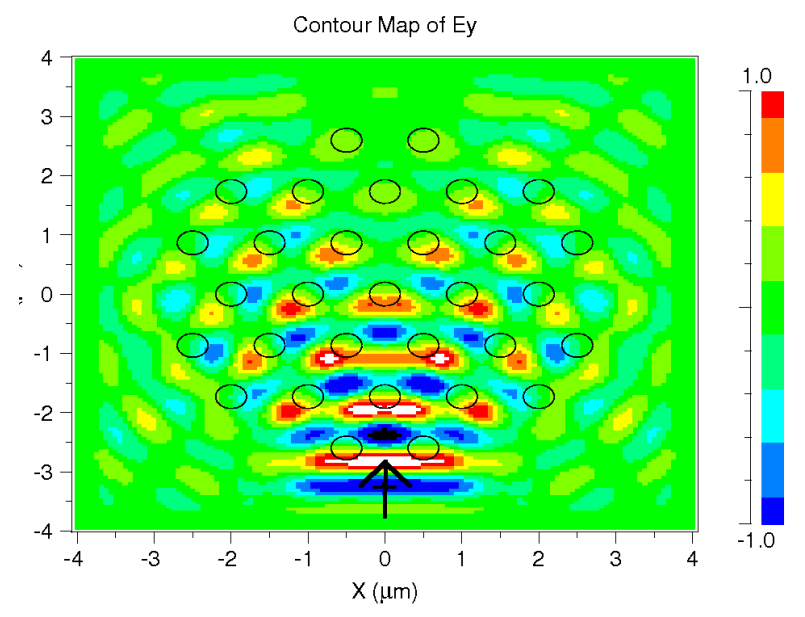

Figure 9. Same finite vortex lattice as in Fig.8, but rotated by $\pi / 6$. A directional band gap is clearly seen as there is no transmission.

\section{CONCLUSION}

An important consequence of such a directional band gap is that it can be utilized to measure the rotation frequency of the BEC. In the vortex lattice experiments, the rotation frequency of the vortex lattice is measured indirectly by inferring it from the radius of the cloud. ${ }^{1-6}$ However, if a directional band gap is established, probing laser beam will be chopped by the rotation of the vortex lattice and counting the chopping frequency will yield a direct measurement of the rotation frequency. Such a measurement can be carried out non-destructively, and continuously. As the rotation frequency of the cloud, which is around hundred Hertz in a typical experiment, is much smaller than the laser frequency, rotation will not affect the optical band structure discussed earlier.

We have shown that photonic band gaps can be generated in vortex lattices of an atomic Bose-Einstein condensate by utilizing quantum coherence based ultra-high index enhancement effect. We considered a two dimensional triangular lattice and found the physical parameter regime for band gaps and other band structure effects to be observed. We argued that the effects found for an infinite lattice could be observed in experiments where tens of vortices are created in the condensate. We finally suggested a method to use a directional band gap to measure the rotation frequency of the condensate non-destructively.

\section{ACKNOWLEDGMENTS}

Ö.E.M. acknowledges support from TUBA-GEBIP Award. M.Ö.O. acknowledges support from TUBITAKKARIYER grant, and thanks E. Özbay for computational support. The authors also acknowledge support by FP6-IST 511616 Network of Excellence PHOREMOST.

\section{REFERENCES}

1. J. R. Abo-Shaeer, C. Raman, J. M. Vogels, and W. Ketterle Science 292, p. 476, 2001.

2. P. C. Haljan, I. Coddington, P. Engels, and E. A. Cornell Phys. Rev. Lett. 87, p. 210403, 2000.

3. K. W. Madison, F. Chevy, W. Wohlleben, and J. Dalibard Phys. Rev. Lett. 84, p. 806, 2000.

4. K. W. Madison, F. Chevy, V. Bretin, and J. Dalibard Phys. Rev. Lett. 86, p. 4443, 2001.

5. N. L. Smith, W. H. Heathcote, J. M. Krueger, and C. J. Foot Phys. Rev. Lett. 93, p. 080406, 2004.

6. P. Engels, I. Coddington, P. C. Haljan, and E. A. Cornell Phys. Rev. Lett. 89, p. 100403, 2002.

7. M. Artoni and I. Carusotto Phys. Rev. A 67, p. 011602(R), 2003. 
8. P. Öhberg Phys. Rev. A 66, p. 021603(R), 2002.

9. E. Yablonovitch, T. J. Gmitter, and K. M. Leung Phys. Rev. Lett. 67, p. 2295, 1991.

10. S. F. A. Mekis and J. D. Joannopoulos Phys. Rev. B 58, p. 4809, 1998.

11. E. Çubukçu, K. Aydın, S. F. E. Özbay, and C. M. Soukoulis Nature(London) 423, p. 604, 2003.

12. M. F. Yanık and S. Fan Phys. Rev. Lett. 92, p. 083901, 2004.

13. L. D. Negro and et al. Phys. Rev. Lett. 90, p. 055501, 2003.

14. E. Yablonovitch Phys. Rev. Lett. 58, p. 2059, 1987.

15. Z. Dutton and J. Ruostekoski Phys. Rev. Lett. 93, p. 193602, 2004.

16. D. Akamatsu and M. Kozuma Phys. Rev. A 67, p. 023803, 2003.

17. A. Mair, A. Vaziri, G. Weihs, and A. Zeilinger Nature (London) 412, p. 313, 2001.

18. H. H. Arnaut and G. A. Barbosa Phys. Rev. Lett. 85, p. 286, 2000.

19. J. Arlt, K. Dholakia, L. Allen, and M. J. Padgett Phys. Rev. A 59, p. 3950, 1999.

20. K.-P. Marzlin and W. Zhang Eur. Phys. J. D 12, p. 241, 2000.

21. T.-L. Ho Phys. Rev. Lett. 87, p. 060403, 2001.

22. M. O. Scully Phys. Rev. Lett. 67, p. 1855, 1991.

23. S. Harris Physics Today 50, p. 36, 1997.

24. C. Liu, Z. Datton, C. H. Behroozi, and L. Hau Nature 409, p. 490, 2001.

25. M. Fleischhauer and M. D. Lukin Phys. Rev. Lett. 84, p. 5094, 2000.

26. M. O. Scully and M. S. Zubairy, Quantum Optics, Cambridge University Press, Cambridge, 1997.

27. M. Fleischhauer, C. Keitel, M. O. Scully, C. Su, B. T. Ulrich, and S.-Y. Zhu Phys. Rev. A 46, p. 1468, 1992.

28. M. Fleischhauer Phys. Rev. A 60, p. 2534, 1999.

29. H. Wallis Phys. Rev. A 56, p. 2060, 1997.

30. O. Morice and et al. Phys. Rev. A 51, p. 3896, 1995.

31. J. Ruestekoski and J. Javanainen Phys. Rev. A 56, p. 2056, 1997.

32. K. V. Krutitsky, K.-P. Marzlin, and J. Audretsch Phys. Rev. A 65, p. 063609, 2002.

33. J. Jackson, Classical Electrodynamics, 2nd ed., Wiley, New York, 1975.

34. M. Cozzini and S. Stringari Phys. Rev. A 67, p. 041602(R), 2003.

35. G. Baym Phys. Rev. Lett. 91, p. 110402, 2003.

36. D. A. Butts and D. S. Rokshar Nature(London) 397, p. 327, 1999.

37. I. Coddington, P. Engels, V. Schweikhard, and E. A. Cornell Phys. Rev. Lett. 91, p. 100402, 2003.

38. M. Ö. Oktel Phys. Rev. A 69, p. 023618, 2004.

39. S. Sinha and G. Shylapnikov cond-mat/04112139, 2004.

40. N. Berloff Jour. Phys. A 37, p. 1617, 2004.

41. F. Chevy and S. Stringari Phys. Rev. A 68, p. 053601, 2003.

42. G. B. G. Watanabe and C. J. Pethick Phys. Rev. Lett. 93, p. 190401, 2004. 